\title{
Autonomous Transition Flight for a Vertical Take-Off and Landing Aircraft
}

\author{
P. Casau, D. Cabecinhas, C. Silvestre \\ Instituto Superior Técnico, Universidade Técnica de Lisboa, Lisboa, Portugal
}

\begin{abstract}
This paper addresses the problem of autonomous transition between hover and level flight for a model-scale fixed-wing Unmanned Air Vehicle (UAV). The UAV system is described as a Hybrid Automaton where the different operating modes correspond to the hover, transition, and level flight regions of the flight envelope. Reference maneuvers are generated so as to provide robustness to the system with respect to exogenous disturbances and parametric uncertainty when performing single operating mode and transition maneuvers. Controllers rendering the closed-loop system Input-to-State Stable (ISS) with restrictions are designed for all three operating modes allowing for practical tracking of the reference maneuvers in the presence of disturbances. Simulation results demonstrate the performance and robustness of the proposed solution.
\end{abstract}

\section{INTRODUCTION}

The demand for Unmanned Air Vehicles (UAVs) has escalated in the past few years due to their contributions in commercial and defense applications, including fire surveillance and mitigation operations, agricultural fields spraying, infrastructure inspection, among others (see e.g. [1], [2], [3], [4] and [5]). Several UAV configurations have been developed to meet the requirements imposed by such applications, including fixed-wing and tilt wing aircraft, rotorcrafts and ductedfan vehicles. Recent developments described in [4], [6] and [7] have shown that fixed-wing Vertical Take-Off and Landing (VTOL) aircrafts can perform both long endurance missions and precise maneuvering within exiguous environments. The versatility of such aircrafts combines helicopter precise trajectory tracking with conventional fixedwing airplanes ability to cover large distances, delivering a final solution which largely exceeds the capabilities of its predecessors. However, the problem of achieving robust transitions between hover and leveled flights is difficult for its exquisite dynamics. To this end, several control methodologies have been employed, including open-loop maneuvers [7], linear optimal techniques [6], locally stable nonlinear controllers [8] and adaptive controllers [9]. In [6], the transition maneuver is performed in open-loop and tested on a experimental setup. This approach however is not systematic and it is not known the extent of its applicability. The work reported in [9] characterizes the aircraft dynamics in terms of several parameters which must be accurately estimated in order to provide aircraft stability. In [8], the transition problem is addressed much more carefully and a path-following algorithm is developed. The work developed in this paper draws inspiration from [8], [6] and [10] and

This work was partially supported by Fundação para a Ciência e a Tecnologia (ISR/IST plurianual funding) through the PIDDAC Program funds and by Project PTDC/MAR/64546/2006 OBSERVFLY. The work of D Cabecinhas and P. Casau was supported with grants SFRH/BD/31439/2006 and SFRH/BD/70656/2010, from Fundação para a Ciência e a Tecnologia. introduces a novel way to tackle the transition between hovered and leveled flight conditions, based on a hybrid control system. The change in aircraft dynamics from hover to level flight suggests that supervisory control is a plausible solution for the given problem. Similar methodologies, like the ones described in [11] and [12], have been successfully employed in a variety of applications. Controller switching during operating mode transitions adds discrete behavior to the continuous UAV model, creating a new layer of complexity that must be dealt appropriately. Systems displaying both continuous and discrete behavior have been under an intense research effort over the last decade. This study has given rise to several concepts such as hybrid automata [10] and switched systems [13] which fall within the broader category of Hybrid Dynamical Systems described in [11]. The discrete behavior built into these systems may appear naturally for certain applications such as UAV landing and take-off (see e.g. [14]) but may also be the consequence of digital control or supervisory control [15].

The solution proposed in this paper employs supervisory control by modeling the small-scale UAV within the Hybrid Automata framework, dividing the aircraft flight envelope into Hover, Transition and Level operating modes. Linear optimal control techniques are employed for system stabilization in Hover and Level Flight while a nonlinear controller that renders the closed-loop system locally Input-to-State Stable (ISS) with restrictions is developed for Transition Flight, enabling practical reference maneuvers tracking with an arbitrarily small tracking error. The nonlinear controller provides robust transition between the two disjoint sets which characterize the Hover and Level operating modes where local stabilization is provided.

This paper is organized as follows. Section II presents the aircraft nonlinear model. Section III introduces the Hybrid Automaton which characterizes the overall system and addresses the reference transition maneuvers. Section IV briefly discusses the local controllers used in hover and level flights, proceeding into a thorough analysis of the nonlinear controller. Finally, simulation results are presented in Section $\mathrm{V}$ making use of the hybrid systems simulator presented in [16].

\section{NONLINEAR UAV MODEL}

The UAV under analysis is the model-scale fixed wing aircraft which has a total of six actuators: $a$ ) 2 Propellers; $b$ ) Rudder; $c$ ) Elevator; $d$ ) Ailerons; $e$ ) Flaps. These actuators are set up in a standard wing/tail configuration (see [18] for a full description of the aerodynamic actuators).

The aircraft dynamics are described in the configuration manifold $\mathbb{S}^{1} \times \mathbb{R}^{2}$ considering that the aircraft motion occurs 


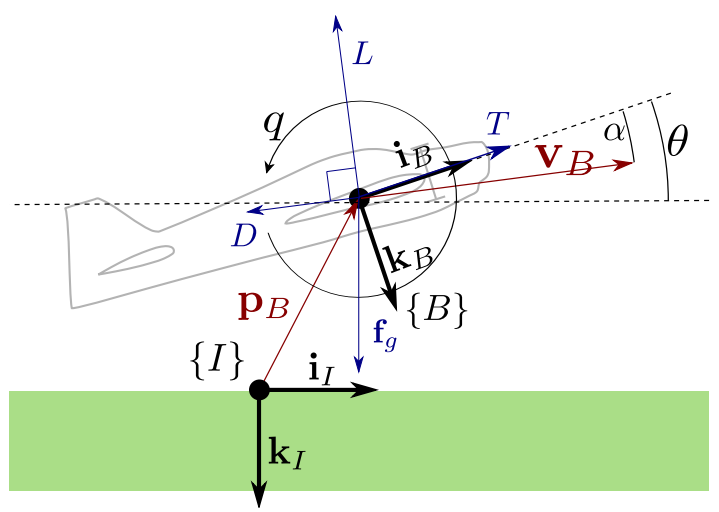

Fig. 1: Planar UAV model representation.

solely on the vertical plane, i.e. the lateral motion is assumed to have a small influence on the longitudinal dynamics. This approach has also been adopted in [8] and [17] because it eases the controller design while not compromising the stability of the three-dimensional system, provided that there exists a lateral controller. In fact, the linear model of an standard airplane flying on the vertical plane has decoupled lateral/longitudinal dynamics (see e.g. [18]), therefore, lateral stabilization can be provided separately by linear control techniques. The actuators influencing the longitudinal motion are the flaps, the elevator and the propellers' common mode (which provides the required thrust). The flap's deflection $\delta_{f} \in\left[\delta_{f_{\text {min }}}, \delta_{f_{\text {max }}}\right]$, the elevator's deflection $\delta_{e} \in$ $\left[\delta_{e_{\min }}, \delta_{e_{\max }}\right]$ and the thrust per propeller $T \in\left[T_{\min }, T_{\max }\right]$ are assumed to be readily available, i.e. the propellers dynamics are much faster than the aircraft dynamics therefore they can be disregarded for control design purposes. Furthermore, the thrust is collinear with the zero-lift line.

The aircraft dynamic model construction requires the definition of an Inertial Reference Frame $\{I\}$ and a Body Reference Frame $\{B\}$ depicted in Figure 1. The reference frame $\{I\}$ is fixed at some point in the Earth's surface which is considered to be flat and still for the current application. It is identified by the set of unitary vectors $\left\{\mathbf{i}_{I}, \mathbf{k}_{I}\right\}$ where $\mathbf{i}_{I}$ is directed to geographic North and is parallel to the ground and $\mathbf{k}_{I}$ is perpendicular to $\mathbf{i}_{I}$ and is directed towards the Nadir. The reference frame $\{B\}$ is identified by the set of unitary vectors $\left\{\mathbf{i}_{B}, \mathbf{k}_{B}\right\}$ which match the principal axis of inertia and has its origin at the vehicle's center of gravity (CG), as represented in Figure 1. For the sake of simplicity, $\mathbf{i}_{B}$ is coincident to the zero-lift line.

The configuration of the body frame $\{B\}$ with respect to $\{I\}$ can be viewed as an element of the Special Euclidean group, $(\mathbf{R}, \mathbf{p}) \in \mathrm{SE}(2)$ where

$$
\mathbf{p}=\left[\begin{array}{ll}
x & z
\end{array}\right]^{\top}, \quad \mathbf{R}=\left[\begin{array}{cc}
\cos \theta & \sin \theta \\
-\sin \theta & \cos \theta
\end{array}\right],
$$

and the rotation matrix is parametrized by the pitch angle $\theta$. The kinematic equations of motion for the aircraft are described by

$$
\dot{\theta}=q, \quad \dot{\mathbf{p}}=\mathbf{R v} .
$$

where $\mathbf{v}=\left[\begin{array}{ll}u & w\end{array}\right]^{\top} \in \mathbb{R}^{2}$ is the linear velocity and $q \in \mathbb{R}$ the angular velocity, expressed both in $\{B\}$ (we borrow the standard aircraft nomenclature described in e.g. [18]).
The forces and moments acting on the aircraft body which affect its behavior are the gravity $\mathbf{f}_{g} \in \mathbb{R}^{2}$, the aerodynamic lift $L \in \mathbb{R}$, the aerodynamic drag $D \in \mathbb{R}$, the thrust $T \in \mathbb{R}$ and the aerodynamic torque $M_{a} \in \mathbb{R}$ which are depicted in Figure 1. The gravity force acting on the aircraft is directed downwards along $\mathbf{k}_{I}$ and it is given by $\mathbf{f}_{g}=[0 \mathrm{mg}]^{\top}$, where $m$ is the vehicle's mass and $g$ is the acceleration of gravity. The aerodynamic force takes into account both lift and drag components which are described by (see e.g. [19])

$$
L_{i}=\frac{1}{2} \rho u_{\infty}^{2} A_{i} C_{L_{i}}(\alpha), \quad D_{i}=\frac{1}{2} \rho u_{\infty}^{2} A_{i} C_{D_{i}}(\alpha),
$$

respectively, where $i$ is the lifting surface identifier ${ }^{1}, u_{\infty}$ denotes the airspeed, $\rho$ denotes the atmospheric pressure, $A$ is the planform area, $C_{L}$ is the Coefficient of Lift and $C_{D}$ is the Coefficient of Drag. These two parameters are dependent on the angle of attack $\alpha=\arctan (w / u)$, the actuator deflection $\delta_{i}$, the maximum angle of attack $\bar{\alpha}$, the surface's aspect ratio $\mathcal{A}$, efficiency $e$ and parasitic coefficient of drag $C_{D_{0}}$ and are given by

$$
\begin{aligned}
C_{L}\left(\alpha, \delta_{i}\right) & = \begin{cases}C_{L_{\alpha}} \alpha+C_{L_{\delta_{i}}} \delta_{i}, & -\bar{\alpha} \leq \alpha \leq \bar{\alpha} \\
0 & \text { otherwise }\end{cases} \\
C_{D}\left(C_{L}\right) & =C_{D_{0}}+\frac{C_{L}^{2}}{\pi \mathcal{A} e} .
\end{aligned}
$$

The coefficient of lift has a linear dependence with the angle of attack and the coefficient of drag has a quadratic dependence with $C_{L}(\alpha)$ for small angles of attack (see e.g. [19]). In general, the actuator deflection $\delta_{i}$ increases the coefficient of lift while reducing the stall angle. This effect should not be noticeable if the aircraft's angle of attack and actuator deflection remain bounded within small values. The aircraft's lifting surfaces (the wing, the horizontal stabilizer and the vertical stabilizer) produce both lift and drag forces, each of which has two contributions: one from the free-stream flow velocity $u_{\infty}=\|\mathbf{v}\|$ and another from the propeller slipstream velocity $u_{\infty}=u_{p}$, given by

$$
u_{p}=\sqrt{8 T / \rho \pi d^{2}},
$$

assuming an inviscid, steady and incompressible flow (see e.g. [21]), being $d$ the propellers' diameter. These two contributions are considered separately and combined together in the end using superposition. These simplifications provide following descriptions of the wing lift $L_{w}$, wing $\operatorname{drag} D_{w}$ and horizontal stabilizer lift $L_{h s}$,

$$
\begin{aligned}
L_{w}= & -\frac{1}{2} \rho A_{w}\|\mathbf{v}\|^{2}\left(C_{L_{\alpha}} \alpha+C_{L_{\delta_{f}}} \delta_{f}\right) \\
& -\frac{1}{2} \rho A_{p, w}\left(2 u_{p}^{2}\right) C_{L_{\delta_{f_{p}, w}}} \delta_{f} \\
D_{w}= & -\frac{1}{2} \rho A_{w}\|\mathbf{v}\|^{2}\left(C_{D_{0_{w}}}+\frac{\left(C_{L_{\alpha}} \alpha+C_{L_{\delta_{f}}} \delta_{f}\right)^{2}}{\pi \mathcal{A}_{w} e_{w}}\right) \\
L_{h s}= & -\frac{1}{2} \rho A_{h s}\|\mathbf{v}\|^{2}\left(C_{L_{\alpha}} \alpha+C_{L_{\delta_{e_{h s}}}} \delta_{e}\right) \\
& -\frac{1}{2} \rho A_{p, h s}\left(2 u_{p}^{2}\right) C_{L_{\delta_{e_{p}, h s}}} \delta_{e}
\end{aligned}
$$

\footnotetext{
${ }^{1}$ The $i$ subscript identifies the source of any given attribute. The wing, horizontal stabilizer, vertical stabilizer and propellers are identified by the letters $w, h s, v s$ and $p$, respectively.
} 
where the subscripts $p, h s$ and $p, w$ have been introduced to distinguish the effects of the free-stream flow and that of the propeller slipstream. Since the main contribution to the aircraft drag is that of the wing, the horizontal and vertical stabilizers drag are not taken into account (this approach yields the positive experimental results reported in [6]). Both the wing lift and the horizontal stabilizer lift are normal to the aircraft velocity while the drag is collinear to it, as depicted in Figure 1. According to this geometry, one may construct the aerodynamic forces along the $x$-axis $\left(X_{a}\right)$ and the $z$-axis $\left(Z_{a}\right)$ in terms of both lift and drag as follows

$$
\left[\begin{array}{c}
X_{a} \\
Z_{a}
\end{array}\right]=-\left[\begin{array}{cc}
\cos \alpha & -\sin \alpha \\
\sin \alpha & \cos \alpha
\end{array}\right]\left[\begin{array}{c}
D_{w} \\
L_{h s}+L_{w}
\end{array}\right] .
$$

The lifting surfaces also create torques which affect the aircraft dynamics because their aerodynamic centers are displaced from the CG along the $x$-axis. The corresponding torques are given by

$$
M_{i}=-x_{a c_{i}} Z_{a_{i}}
$$

where $i$ is the surface identifier and $x_{a c}$ is the aerodynamic center location along the $x$-axis of the $\{B\}$ frame. There is also a damping torque $M_{\text {damp }}$ which arises due to slipstream lag and also an angle of attack increase in the tail due to rotation. The stabilizing moment produced due to these effects is described by the relation

$$
\begin{aligned}
M_{d a m p}= & -\frac{1}{2} \rho A_{p, h s} C_{L_{\alpha_{h s}}} q\left(x_{p}-x_{a c_{h s}}\right)\left|x_{a c_{h s}}\right| 2 u_{p} \\
& -\frac{1}{2} \rho A_{h s} C_{L_{\alpha_{h s}}} x_{a c_{h s}}^{2} q u
\end{aligned}
$$

given in [6] and [18], where $x_{p}$ is the propellers' location.

Having defined the reference frames $\{I\}$ and $\{B\}$, their relations and the forces acting upon the system, the aircraft dynamic model is derived from newton equations of motion (see e.g. [17]) and it is given by

$$
\begin{aligned}
& \dot{u}=\frac{X_{a}+2 T}{m}-g \sin \theta-q w+\delta_{u}(t), \\
& \dot{w}=\frac{Z_{a}}{m}+g \cos \theta+q u+\delta_{w}(t), \\
& \dot{q}=\frac{M_{h s}+M_{w}+M_{d a m p}}{J}+\delta_{q}(t),
\end{aligned}
$$

where $J$ is the aircraft's moment of inertia, $\delta_{u}(t), \delta_{w}(t)$ and $\delta_{q}(t)$ denote unknown perturbations which may appear due to parametric uncertainty, external disturbances and/or due to deviations from the vertical plane.

The analysis of (5) reveals two very distinct regions of operation: the leveled flight operative mode and the hovered flight operative mode. In the former, it is the wing lift which counteracts the gravity force whilst, in the latter, it is the propellers' thrust. It should be clear that the aerodynamic surfaces always produce some actuation except when $T=0$ and $\mathbf{v}=0$. This is a very unusual situation which does not happen in any of the aircraft operative modes discussed from hereon.

The next section builds the system's Hybrid Automaton resulting from the switching between operative modes, taking into consideration the underlying framework and the mission goals.

\section{HYBRID AUTOMATON}

The system's discrete behavior is captured by means of a Hybrid Automaton which is identified by: a set of the operating modes $\mathcal{Q}$; a domain mapping $\mathcal{D}: \mathcal{Q} \rightrightarrows \mathbb{R}^{n} \times \mathbb{R}^{m}$; a flow map $f: \mathcal{Q} \times \mathcal{D} \rightarrow \mathbb{R}^{n}$; a set of edges $\mathcal{E} \subset \mathcal{Q} \times \mathcal{Q}$; a guard mapping $\mathcal{G}: \mathcal{E} \rightrightarrows \mathbb{R}^{n} \times \mathbb{R}^{m}$ and; a reset map $\mathcal{R}: \mathcal{E} \times \mathbb{R}^{n} \times \mathbb{R}^{m} \rightarrow \mathbb{R}^{n}$. The system state $\boldsymbol{\xi} \in \mathbb{R}^{6}$ and the actuator input $\boldsymbol{\mu} \in \mathbb{R}^{3} \times \mathcal{Q}^{\star}$ are given by

$$
\boldsymbol{\xi}=\left[\begin{array}{llllll}
u & w & q & \theta & x & z
\end{array}\right]^{\top}, \quad \boldsymbol{\mu}=\left[\begin{array}{llll}
T & \delta_{f} & \delta_{e} & q^{\star}
\end{array}\right]^{\top},
$$

where a new input variable $q^{\star} \in \mathcal{Q}^{\star}$ is required to inform the controller which is the desired operating mode and whether transition is required. The remaining state and input variables are defined in Section II. The Hybrid Automaton representation is provided in Figure 2.

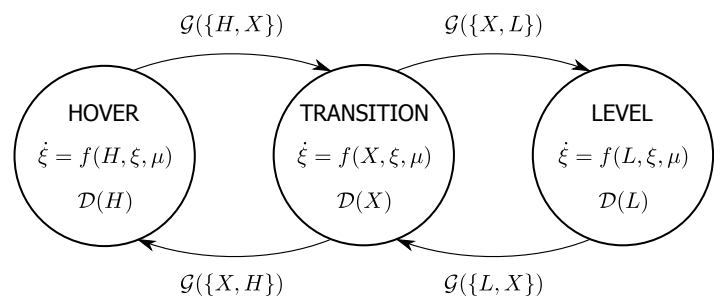

Fig. 2: UAV Hybrid Automaton diagram.

In this Hybrid Automaton, the flight envelope is divided into Hover, Level and Transition operative modes. In short, the overall system is stable if: $a$ ) there exist local controllers which stabilize the aircraft in Hover and Level operative modes; $b$ ) the transition trajectory remains $\epsilon$-far from the domain limits, and; c) the transition controller is able to perform practical trajectory tracking with arbitrarily small tracking error. The reader is referred to [10] and [11] for further details on the Hybrid Automaton framework.

The reference transition trajectories are computed by means of nominal system inversion, verify $|\alpha(t)|<|\bar{\alpha}|$ and are identified by

$$
\begin{aligned}
& \boldsymbol{\xi}^{\star}(t)=\left[u^{\star}(t) w^{\star}(t) q^{\star}(t) \theta^{\star}(t) x^{\star}(t) z^{\star}(t)\right]^{\top}, \\
& \boldsymbol{\mu}^{\star}(t)=\left[\begin{array}{llll}
T^{\star}(t) & \delta_{f}^{\star}(t) & \delta_{e}^{\star}(t) & q^{\star}
\end{array}\right]^{\top} \text {. }
\end{aligned}
$$

\section{Controller Design}

The controller design comprises two different methods: linear optimal control techniques are used when in Hover or Level operating modes, providing local stabilization; nonlinear control is used to perform the transition between the two disjoint operating modes.

Classic Linear Quadratic Regulator (LQR) techniques provide the full state feedback control law $\tilde{\boldsymbol{\mu}}=-\mathbf{K} \tilde{\xi}$ which robustly stabilizes the aircraft within a sublevel set of the Lyapunov function $V(\tilde{\boldsymbol{\xi}})=\tilde{\boldsymbol{\xi}}^{T} \tilde{\boldsymbol{\xi}}$ near the linearization point $\left(\boldsymbol{\xi}_{0}, \boldsymbol{\mu}_{0}\right)$, where $\tilde{\boldsymbol{\xi}}=\boldsymbol{\xi}-\boldsymbol{\xi}_{0}$ and $\tilde{\boldsymbol{\mu}}=\boldsymbol{\mu}-\boldsymbol{\mu}_{0}$.

In order to improve the dynamic model clarity and simplify the nonlinear controller design we propose a redefinition of the control inputs as follows. The lift forces $L_{e}$ and $L_{f}$ produced upon actuator deflection are related to the inputs $\delta_{e}$ and $\delta_{f}$ according to

$$
L_{e}=-\delta_{e}\left(\frac{1}{2} \rho\left(2 u_{p}^{2}\right) A_{p, h s} C_{L_{\delta_{e_{p}, h s}}}+\frac{1}{2} \rho\left\|\mathbf{v}_{B}\right\|^{2} A_{h s} C_{L_{\delta_{e_{h s}}}}\right),
$$




$$
L_{f}=-\delta_{f}\left(\frac{1}{2} \rho\left(2 u_{p}^{2}\right) A_{p, w} C_{L_{\delta_{p}, w}}+\frac{1}{2} \rho\left\|\mathbf{v}_{B}\right\|^{2} A_{w} C_{L_{\delta_{f}}}\right) .
$$

The new control inputs $\tau_{u}, \tau_{w}$ and $\tau_{q}$ are related to the real control inputs by

$$
\left[\begin{array}{c}
\tau_{u} \\
\tau_{w} \\
\tau_{q}
\end{array}\right]=\left[\begin{array}{ccc}
\frac{2}{m} & 0 & 0 \\
0 & \frac{1}{m} & \frac{1}{m} \\
0 & -\frac{x_{a c_{h s}}}{I} & -\frac{x_{a c_{w}}}{I}
\end{array}\right]\left[\begin{array}{ccc}
1 & \sin \alpha & \sin \alpha \\
0 & \cos \alpha & 0 \\
0 & 0 & \cos \alpha
\end{array}\right]\left[\begin{array}{l}
T \\
L_{e} \\
L_{f}
\end{array}\right] .
$$

Substituting (6), (3), (4) and (2) into (1) and (5) we get

$$
\begin{aligned}
\dot{u} & =\tau_{u}+h_{u}(u, w, q, \theta)+\delta_{u}(t), \\
\dot{w} & =\tau_{w}+h_{w}(u, w, q, \theta)+\delta_{w}(t), \\
\dot{q} & =\tau_{q}+h_{q}(u, w, q),+\delta_{q}(t), \\
\dot{\theta} & =q,
\end{aligned}
$$

where $h_{u}: \mathbb{R}^{4} \rightarrow \mathbb{R}, h_{w}: \mathbb{R}^{4} \rightarrow \mathbb{R}$ and $h_{q}: \mathbb{R}^{3} \rightarrow \mathbb{R}$ collect the dynamics which do not depend on the actuator input. This substitution effectively rescales the control input throughout the maneuver. The proposed controller for system (7) is described by

$$
\boldsymbol{\mu}=\left[\begin{array}{cl}
\tau_{u}^{\star}(t)+\tilde{\tau_{u}} \\
\tau_{w}^{\star}(t)+\tilde{\tau_{w}} \\
\tau_{q}^{\star}(t)+\tilde{\tau_{q}}
\end{array}\right], \quad \begin{aligned}
\tilde{\tau_{q}}=-k_{u} \tilde{u} \\
\tilde{\tau_{w}}=-k_{w} \tilde{w}
\end{aligned}
$$

where $\tau_{u}^{\star}(t), \tau_{q}^{\star}(t)$ and $\tau_{w}^{\star}(t)$ from $T^{\star}(t), \delta_{e}^{\star}(t)$ and $\delta_{f}^{\star}(t)$ using the relation (6). Proportional-derivative (PD) controllers are used to track the reference trajectories. Substituting (8) into the system state equations (7), the aircraft error dynamics are described by

$$
\begin{aligned}
\dot{\tilde{u}} & =\tilde{\tau_{u}}+\Psi_{u}(\tilde{u}, \tilde{w}, \tilde{q}, \tilde{\theta}, t)+\delta_{u}(t), \\
\dot{\tilde{w}} & =\tilde{\tau_{w}}+\Psi_{w}(\tilde{u}, \tilde{w}, \tilde{q}, \tilde{\theta}, t)+\delta_{w}(t), \\
\dot{\tilde{q}} & =\tilde{\tau_{q}}+\Psi_{q}(\tilde{u}, \tilde{w}, \tilde{q}, t)+\delta_{q}(t), \\
\dot{\tilde{\theta}} & =\tilde{q},
\end{aligned}
$$

where the functions $\Psi_{u}, \Psi_{w}$ and $\Psi_{q}$ are given by

$$
\begin{aligned}
\Psi_{u}(\tilde{u}, \tilde{w}, \tilde{q}, \tilde{\theta}, t)= & h_{u}\left(u^{\star}(t)+\tilde{u}, w^{\star}(t)+\tilde{w}, q^{\star}(t)+\tilde{q}, \theta+\tilde{\theta}\right) \\
& -h_{u}\left(u^{\star}(t), w^{\star}(t), q^{\star}(t), \theta^{\star}(t)\right) \\
\Psi_{w}(\tilde{u}, \tilde{w}, \tilde{q}, \tilde{\theta}, t)= & h_{w}\left(u^{\star}(t)+\tilde{u}, w^{\star}(t)+\tilde{w}, q^{\star}(t)+\tilde{q}, \theta+\tilde{\theta}\right) \\
& -h_{w}\left(u^{\star}(t), w^{\star}(t), q^{\star}(t), \theta^{\star}(t)\right) \\
\Psi_{q}(\tilde{u}, \tilde{w}, \tilde{q}, t)= & h_{q}\left(u^{\star}(t)+\tilde{u}, w^{\star}(t)+\tilde{w}, q^{\star}(t)+\tilde{q}\right) \\
& -h_{q}\left(u^{\star}(t), w^{\star}(t), q^{\star}(t)\right) .
\end{aligned}
$$

The reference trajectory is one of equilibrium, in the absence of disturbances, because

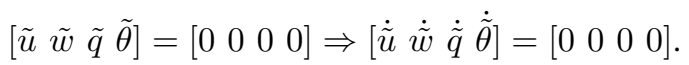

The previous set of equations provides the foundations upon which the nonlinear controller's robustness emerges. Consider two separate but interconnected systems which describe the pairs $(\tilde{u}, \tilde{w})$ and $\left(\theta_{1}, \theta_{2}\right)$, where $\theta_{1}=\tilde{\theta}$ and $\theta_{2}=\tilde{q}+\frac{\tilde{\theta}}{k_{q}}$. Input-to-State Stability is proven firstly for each of these systems separately in Propositions 1 and 2. Input-toState Stability for the overall system then follows from the small gain theorem described in both [22] and [23], which is applied to the feedback interconnection. The conditions of the small gain theorem hold because it is possible to set the input gains arbitrarily small with a proper choice of the controller gains.
Proposition 1: For some $c_{u}^{\star}>0$ and $c_{w}^{\star}>0$ and any numbers satisfying $\Delta>0, \Delta_{\theta}>0,0<c_{u}<c_{u}^{\star}$ and $0<c_{w}<c_{w}^{\star}$ there exist $k_{u} \geq k_{u}^{\star}$ and $k_{w} \geq k_{w}^{\star}$ such that the system with the dynamics (9a) and (9b) is rendered ISS with restrictions $c_{u}$ in the initial state $\tilde{u}(0), c_{w}$ on the initial state $\tilde{w}(0), \Delta$ on the inputs $\delta_{u}(t)$ and $\delta_{w}(t)$ and $\Delta_{\theta}$ on the input $\left(\theta_{1}(t), \theta_{2}(t)\right)$.

Proof: Consider the Lyapunov function (11) and the level set definition given in (12).

$$
\begin{gathered}
V_{1}(\tilde{u}, \tilde{w})=\frac{1}{2}\left(\tilde{u}^{2}+\tilde{w}^{2}\right) \\
\Omega_{1}(l)=\left\{(\tilde{u}, \tilde{w}) \in \mathbb{R}^{2}: V_{1}(\tilde{u}, \tilde{w}) \leq l\right\}
\end{gathered}
$$

It turns out that, due to radial unboundedness there exist positive $l_{1}$ such that

$$
\left\{(\tilde{u}, \tilde{w}) \in \mathbb{R}^{2}:|\tilde{u}| \leq c_{u} \wedge|\tilde{w}| \leq c_{w}\right\} \subset \Omega_{1}\left(l_{1}\right) .
$$

Moreover, for any given reference trajectory it is possible to find $c_{u}^{\star}, c_{w}^{\star}$ and $l_{1}^{\star}$ such that

$$
\left\{(\tilde{u}, \tilde{w}) \in \mathbb{R}^{2}:|\tilde{u}| \leq c_{u}^{\star} \wedge|\tilde{w}| \leq c_{w}^{\star}\right\} \subset \Omega_{1}\left(l_{1}^{\star}\right),
$$

and

$$
\left\{(\tilde{u}, \tilde{w}) \in \Omega_{1}\left(l_{1}^{\star}\right): u^{\star}(t)+\tilde{u}>0 \wedge\left|\arctan \left(\frac{w^{\star}(t)+\tilde{w}}{u^{\star}(t)+\tilde{u}}\right)\right|<\bar{\alpha}\right\},
$$

hold true for all $t \geq 0$.

The functions defined in (10) are locally Lipschitz because the functions $h_{u}, h_{w}$ and $h_{q}$ are continuous and proper, therefore there exist positive $\Gamma_{u}$ and $\Gamma_{w}$ such that for all $(\tilde{u}, \tilde{w}) \in \Omega_{1}\left(l_{1}\right)$ and $\left\|\left(\theta_{1}(t), \theta_{2}(t)\right)\right\|_{\infty}<\Delta_{\theta}$ the following holds

$$
\begin{aligned}
& \left\|\Psi_{u}\left(\tilde{u}, \tilde{w}, \theta_{2}-\frac{\theta_{1}}{k_{q}}, \theta_{1}, t\right)\right\| \leq \Gamma_{u}\left\|\left(\tilde{u}, \tilde{w}, \theta_{1}, \theta_{2}, t\right)\right\|, \\
& \left\|\Psi_{w}\left(\tilde{u}, \tilde{w}, \theta_{2}-\frac{\theta_{1}}{k_{q}}, \theta_{1}, t\right)\right\| \leq \Gamma_{w}\left\|\left(\tilde{u}, \tilde{w}, \theta_{1}, \theta_{2}, t\right)\right\|,
\end{aligned}
$$

for all $t \geq 0$. It can be shown that the Lyapunov function derivative $\dot{V}_{1}$ is upper bounded by

$$
\begin{aligned}
\dot{V}_{1} \leq & -\lambda_{\min }(\mathbf{M})\|(\tilde{u}, \tilde{w})\|^{2}+ \\
& +\|(\tilde{u}, \tilde{w})\|\left(\left(\Gamma_{u}+\Gamma_{w}\right)\left\|\left(\theta_{1}, \theta_{2}\right)\right\|+\right. \\
& \left.+\delta_{u}(t)+\delta_{w}(t)\right),
\end{aligned}
$$

where $\lambda_{\min }(\mathbf{M})>0$ is the smallest eigenvalue of the matrix $\mathbf{M}$, given by

$$
\mathbf{M}=\left[\begin{array}{cc}
k_{u}-\Gamma_{u} & \frac{1}{2}\left(\Gamma_{u}+\Gamma_{w}\right) \\
\frac{1}{2}\left(\Gamma_{u}+\Gamma_{w}\right) & k_{w}-\Gamma_{w}
\end{array}\right] .
$$

Then, it is straightforward to verify that for any $\Delta>0$ and $\Delta_{\theta}>0$ there exist $k_{w}^{\star}>0$ and $k_{u}^{\star}\left(k_{w}^{\star}\right)>0$ such that for any $k_{u} \geq k_{u}^{\star}, k_{w} \geq k_{w}^{\star},\left(\delta_{u}(t), \delta_{w}(t)\right)$ satisfying $\left\|\delta_{u}(t)\right\|_{\infty} \leq \Delta$, $\left\|\delta_{w}(t)\right\|_{\infty} \leq \Delta$, and $\left\|\left(\theta_{1}(t), \theta_{2}(t)\right)\right\|_{\infty} \leq \Delta_{\theta}$ and for any $(\tilde{u}, \tilde{w}) \in \Omega_{1}\left(l_{1}\right)$ the following holds

$$
\dot{V}_{1}<0 \text { if }\|(\tilde{u}, \tilde{w})\|>\frac{\Gamma_{u}+\Gamma_{w}}{\lambda_{\min }(\mathbf{M})}\left\|\left(\theta_{1}(t), \theta_{2}(t)\right)\right\|+\frac{\delta_{u}(t)+\delta_{w}(t)}{\lambda_{\min }(\mathbf{M})} .
$$

The system has a local ISS-Lyapunov function, therefore it is ISS with restrictions $c_{u}$ on the initial state $\tilde{u}(0), c_{w}$ on the initial state $\tilde{w}(0)$ and $\Delta$ on the inputs $\delta_{u}(t)$ and $\delta_{w}(t)$ as long as the conditions $c_{u}<c_{u}^{\star}$ and $c_{w}<c_{w}^{\star}$ are satisfied. 
Proposition 2 employs similar arguments to those in Proposition 1 proof in order to justify the Input-to-State Stability of the closed-loop system $\left(\theta_{1}, \theta_{2}\right)$.

Proposition 2: For any arbitrary positive numbers $\Delta, \Delta_{V}$, $k_{q}, c_{q}$ and $c_{\theta}$ there exists $k_{\theta}^{\star}\left(k_{q}\right)>0$ such that $k_{\theta}>k_{\theta}^{\star}$ renders the system with the dynamics (9c) and (9d) ISS with restrictions $c_{q}$ on the initial state $\tilde{q}(0), c_{\theta}$ on the initial state $\tilde{\theta}(0), \Delta_{V}$ on the input $(\tilde{u}(t), \tilde{w}(t))$ and $\Delta$ on the input $\delta_{q}(t)$.

Proof: Consider the Lyapunov function described by

$$
V_{2}\left(\theta_{1}, \theta_{2}\right)=\frac{1}{2}\left(\theta_{1}^{2}+\theta_{2}^{2}\right)
$$

and the level set definition given by

$$
\Omega_{2}(l)=\left\{\left(\theta_{1}, \theta_{2}\right) \in \mathbb{R}^{2}: V_{2}\left(\theta_{1}, \theta_{2}\right) \leq l\right\}
$$

Due to radial unboundedness, there exists positive $l_{2}$ such that

$$
\left\{\left(\theta_{1}, \theta_{2}\right) \in \mathbb{R}^{2}:\left|\theta_{1}\right| \leq c_{\theta} \wedge\left|\theta_{2}-\frac{\theta_{1}}{k_{q}}\right| \leq c_{q}\right\} \subset \Omega_{2}\left(l_{2}\right) .
$$

The function $\Psi_{q}$ defined in (10) is locally Lipschitz in $\Omega_{2}\left(l_{2}\right)$ because $h_{q}$ is continuous and proper, therefore there exists positive $L_{q}$ such that for all $\left(\theta_{1}, \theta_{2}\right) \in \Omega_{2}\left(l_{2}\right)$, $\|(\tilde{u}(t), \tilde{w}(t))\|_{\infty}<\overline{\|(\tilde{u}, \tilde{w})\|}$ the following holds

$$
\left\|\Psi_{q}\left(\tilde{u}, \tilde{w}, \theta_{2}-\frac{\theta_{1}}{k_{q}}, t\right)\right\| \leq \Gamma_{q}\left\|\left(\tilde{u}, \tilde{w}, \theta_{1}, \theta_{2}, t\right)\right\|,
$$

uniformly for all $t \geq 0$. For any $\varsigma>0$ it is possible to find the following upper bound on the Lyapunov function derivative $\dot{V}_{2}$

$$
\begin{aligned}
\left.\dot{V}_{2}\right|_{\Omega_{2}\left(l_{2}\right)} & \leq-\left(\frac{1}{k_{q}}-\frac{\zeta}{2}\left(1+\Gamma_{q}+\frac{1}{k_{q}^{2}}\right)\right) \theta_{1}^{2} \\
& -\left(k_{\theta} k_{q}-\frac{1}{k_{q}}-\Gamma_{q}-\frac{1}{2 \zeta}\left(1+\Gamma_{q}+\frac{1}{k_{q}^{2}}\right)\right) \theta_{2}^{2} \\
& +\left|\theta_{2}\right|\left(\left|\delta_{q}\right|+\Gamma_{q}\|(\tilde{u}, \tilde{w})\|\right) .
\end{aligned}
$$

For any $k_{q}>0$ and $\lambda>1 / k_{q}$, the choice of parameters $\zeta \leq \zeta^{\star}\left(k_{q}\right)$ and $k_{\theta} \geq k_{\theta}^{\star}\left(\zeta, k_{q}\right)$ with

$$
\begin{aligned}
\zeta^{\star} & =\frac{2}{1+\Gamma_{q}+\frac{1}{k_{q}^{2}}}\left(\lambda-\frac{1}{k_{q}}\right), \text { and } \\
k_{\theta}^{\star} & =\frac{1}{k_{q}}\left(\lambda+\frac{1}{k_{q}}+\Gamma_{q}+\frac{1}{2 \zeta}\left(1+\Gamma_{q}+\frac{1}{k_{q}^{2}}\right)\right),
\end{aligned}
$$

provides the following upper bound on the Lyapunov function derivative

$$
\left.\dot{V}_{2}\right|_{\Omega_{2}\left(l_{2}\right)} \leq-\lambda\left\|\left(\theta_{1}, \theta_{2}\right)\right\|^{2}+\left\|\left(\theta_{1}, \theta_{2}\right)\right\|\left(\left|\delta_{q}\right|+\Gamma_{q}\|(\tilde{u}, \tilde{w})\|\right) .
$$

Therefore, the system has a local ISS Lyapunov function with asymptotic gain $\lambda^{-1} \in \mathbb{R}$. The restrictions on the inputs $\left\|\delta_{q}(t)\right\|_{\infty} \leq \Delta$ and $\|(\tilde{u}(t), \tilde{w}(t))\|_{\infty} \leq \Delta_{V}$ can be set arbitrarily large because the asymptotic gain can be made arbitrarily small. Thus, the proposition holds.

Notice that the restrictions on the inputs of the interconnected systems

$$
\|(\tilde{u}(t), \tilde{w}(t))\|_{\infty} \leq \Delta_{V} \text { and }\left\|\left(\theta_{1}(t), \theta_{2}(t)\right)\right\|_{\infty} \leq \Delta_{\theta}
$$

are satisfied by taking

$$
\Delta_{V}=\max _{(\tilde{u}, \tilde{w}) \in \Omega_{1}\left(l_{1}\right)}\|(\tilde{u}, \tilde{w})\|, \quad \Delta_{\theta}=\max _{\left(\theta_{1}, \theta_{2}\right) \in \Omega_{2}\left(l_{2}\right)}\left\|\left(\theta_{1}, \theta_{2}\right)\right\| .
$$

Under the previous definitions and results, the input-to-state stability for the overall system is established in Proposition 3.

Proposition 3: For some $c_{u}^{\star}>0$ and $c_{w}^{\star}>0$ and any numbers satisfying $\Delta>0,0<c_{u}<c_{u}^{\star}$, $0<c_{w}<c_{w}^{\star}$, $k_{q}>0, c_{q}>0$ and $c_{\theta}>0$ there exist $k_{u}^{\star}>0, k_{w}^{\star}>0$ and $k_{\theta}^{\star}\left(k_{q}\right)>0$ such that the system with dynamics (9) is rendered ISS with restrictions $c_{u}$ on the initial state $\tilde{u}(0), c_{w}$ on the initial state $\tilde{w}(0), c_{q}$ on the initial state $\tilde{q}(0), c_{\theta}$ on the initial state $\tilde{\theta}(0)$ and $\Delta$ on the inputs $\delta_{u}(t), \delta_{w}(t)$ and $\delta_{q}(t)$.

Proof: Input-to-state stability with restrictions for the individual systems $(\tilde{u}, \tilde{w})$ and $\left(\theta_{1}, \theta_{2}\right)$ is proved in Propositions 1 and 2, respectively. The small gain theorem requires that the condition

$$
k_{1} k_{2}<1
$$

is met, where $k_{1}$ is the closed-loop system $(\tilde{u}, \tilde{w})$ asymptotic gain relative to the input $\left(\theta_{1}, \theta_{2}\right)$ and, similarly, $k_{2}$ is the closed-loop system $\left(\theta_{1}, \theta_{2}\right)$ asymptotic gain relative to the input $(\tilde{u}, \tilde{w})$. The asymptotic gain $k_{1}$ decreases with increasing $k_{u}$ or $k_{w}$ and $k_{2}$ can be fixed arbitrarily with an appropriate choice of $k_{\theta}$, therefore, the small gain theorem condition is met. Moreover, the tracking error can be made arbitrarily small.

Proposition 3 concludes the stability and robustness analysis. We have proven that a transition trajectory can be tracked with an arbitrary small error. This allows for the use of the Hybrid Automata framework to achieve stability of the overall hybrid system. The next section presents simulation results which makes use of the open-source hybrid systems simulator presented in [16].

\section{Simulation Results}

The simulations were performed using the open-source tool provided in [16] employing the hybrid automaton equivalence to the generic hybrid system which is given in [11]. These simulations require the definition of the reference maneuvers and of the quantities which define the guard and the domain maps. These quantities are $\bar{\alpha}=15^{\circ}, T_{\min }=0$ $\mathrm{N}, T_{\max }=13 \mathrm{~N}, \delta_{f_{\max }}=7.5^{\circ}$ and $\delta_{e_{\max }}=15^{\circ}$. It is noticeable that the flaps' maximum deflection is half of the elevators', because the flaps and the ailerons share the same surface. Moreover, the quantities $\delta_{f_{m i n}}$ and $\delta_{e_{m i n}}$ are symmetric to their maximum counterparts.

The reference transition trajectories for the transition maneuvers employed in the simulation meet the restriction identified in Section III. The simulation environment includes the influence of atmospheric turbulence, given by the VonKarman turbulence model described in [24] considering a low turbulence scenario. The simulations also require the definition of the controller gains $k_{u}=k_{w}=10 \mathrm{~N} . \mathrm{s} / \mathrm{m}$, $k_{q}=1 \mathrm{~s}$ and $k_{\theta}=10 \mathrm{~N} . \mathrm{s} / \mathrm{m}$. The remaining aircraft parameters are $m=1.64 \mathrm{~kg}, I=0.08 \mathrm{~kg} \cdot \mathrm{m}^{2}, A_{w}=0.29$ $\mathrm{m}^{2}, \mathcal{A}_{w}=3.89, e_{w}=0.8, C_{D_{0 w}}=0.01, x_{a c_{w}}=0.03 \mathrm{~m}$, $A_{p, w}=0.066 \mathrm{~m}^{2}, C_{L_{\alpha_{w}}}=4.07 \mathrm{rad}^{-1}, C_{L_{\delta_{f}, w}}=3.63$, $C_{L_{\delta_{w}}}=2.88, A_{h s}=0.0575 \mathrm{~m}^{2}, x_{a c_{h s}}=-0.56 \mathrm{~m}$, $A_{p, h s}=0.0155 \mathrm{~m}^{2}, C_{L_{\alpha_{h s}}}=4.07, C_{L_{\delta_{e_{p}, h s}}}=4.07$ and $C_{L_{\delta_{h s}}}=4.07, d=0.23 \mathrm{~m}, x_{p}=0.1 \mathrm{~m}$.

Figures 3, 4 and 5 depict the aircraft behavior in terms of state and input variables. Moreover, the references maneuvers 
are also depicted in dashed lines. The aircraft starts in hover at $u=0 \mathrm{~m} / \mathrm{s}$ and the local controller increases thrust in order to achieve a forward velocity of $u=1 \mathrm{~m} / \mathrm{s}$ which is the transition maneuver starting point. When the aircraft state is near $v_{X \rightarrow H}^{\star}(0)$ a switch occurs (at $t \simeq 0.83 \mathrm{~s}$ ) and the nonlinear controller is used in order to follow the reference. Transition to level flight occurs when $\theta<13^{\circ}$ (at $t=8.2 \mathrm{~s}$ ) and the local regulator stabilizes the aircraft at the equilibrium point. The tracking error lies within the level sets $\Omega_{1}\left(l_{1}\right)$ and $\Omega_{2}\left(l_{2}\right)$ therefore there exists a robust transition between the Transition and Level operating modes.

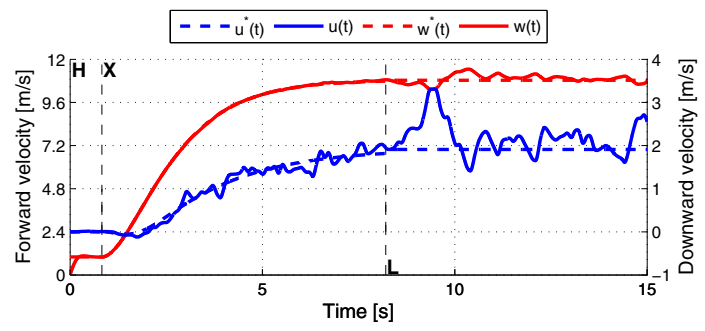

Fig. 3: Transition from hover to level flight - forward velocity $u$ and downward velocity $w$.

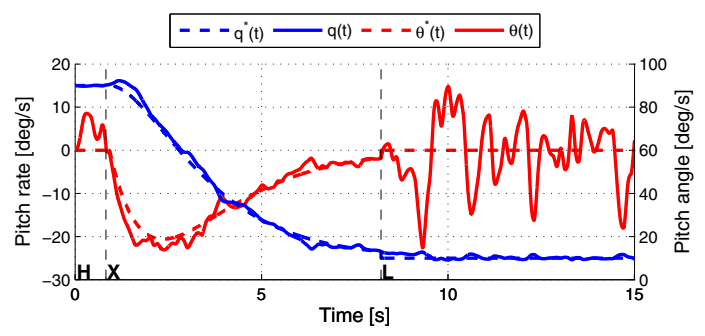

Fig. 4: Transition from hover to level flight - pitch angle $\theta$ and pitch rate $q$.

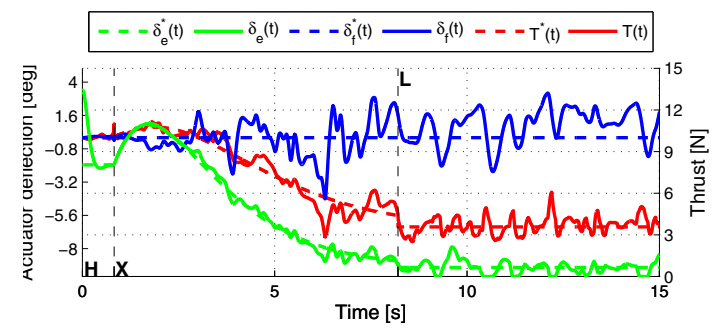

Fig. 5: Transition from hover to level flight - Elevator lift $L_{e}$ and flap lift $L_{f}$ (left axis) and thrust $T$ (right side).

\section{CONCLUSIONS}

This paper presented a solution for the problem of autonomous transition between hover and level flight for a model-scale fixed-wing Unmanned Air Vehicle (UAV). The flight envelope was partitioned in different regions corresponding to hover, transition, and level flight and a Hybrid Automaton model for the overall system was then designed where the operating modes correspond to the different flight envelope regions.
Reference maneuvers were generated so as to provide system robustness against exogenous disturbances and parametric uncertainty when performing single operating mode and transition maneuvers. Controllers rendering the closedloop system Input-to-State Stable with restrictions were designed for all three operating modes allowing for practical tracking of the reference maneuvers with arbitrarily small tracking errors. Simulation results attested the performance and robustness of the proposed solution.

These achievements constitute a novel way to perform controlled transition between hover and level flights for a VTOL aircraft. Trajectory optimization may be performed in order to reduce the control effort and the time for transition.

\section{REFERENCES}

[1] Civilian applications: the challenges facing the uav industry. Air \& Space Europe, 1999.

[2] T. Samad, J. Bay, and D. Godbole. Network-centric systems for military operations in urban terrain: The role of uavs. In Proceedings of the IEEE, 2007.

[3] G. Zhou and D. Zang. Uav system for earth observation. Geoscience and Remote Sensing Symposium, 2007.

[4] W.E. Green and P.Y. Oh. A mav that flies like an airplane and hovers like a helicopter. In Advanced Intelligent Mechatronics. Proceedings, 2005 IEEE/ASME International Conference on, pages 693-698, 2005.

[5] K. Ro, J. S. Oh, and L. Dong. Lessons learned: Application of small uav for urban highway traffic monitoring. In 45th AIAA Aerospace Sciences Meeting and Exhibit, 2007.

[6] Frank Adrian, James S. McGrew, Mario Valenti, Daniel Levine, and Jonathan P. How. Hover, transition, and level flight control design for a single-propeller indoor airplane. In AIAA Guidance, Navigation and Control Conference, 2007.

[7] Alexis Lussier Desbiens, Alan Asbeck, and Mark Cutkosky. Hybrid aerial and scansorial robotics. In IEEE Conference on Robotics and Automation, 2010.

[8] Roberto Naldi and Lorenzo Marconi. On robust transition maneuvers for a class of tail-sitter vehicles. In Proceedings of the Conference on Decision and Control, 2010.

[9] N.B. Knoebe and T.W. McLain. Adaptive quaternion control of a miniature tailsitter uav. In American Control Conference, 2008, pages $2340-2345,2008$.

[10] L. Marconi, R. Naldi, and L. Gentili. A control framework for robust practical tracking of hybrid automata. In Joint 48th IEEE Conference on Decision and Control and 28th Chinese Control Conference, 2009.

[11] Rafal Goebel, Ricardo G. Sanfelice, and Andrew R. Teel. Hybrid dynamical systems. IEEE Control Systems Magazine, 2009.

[12] Ricardo G. Sanfelice and Andrew R. Teel. A throw-and-catch hybrid control strategy for robust global stabilization of nonlinear system. In American Control Conference, 2007.

[13] Daniel Liberzon and Stephen Morse. Basic problems in stability and design of switched systems. IEEE Control Systems Magazine, 1999.

[14] D. Cabecinhas, R. Naldi, L. Marconi, C. Silvestre, and R. Cunha. Robust take-off and landing for a quadrotor vehicle. In IEEE Conference on Robotics and Automation, 2010.

[15] Xenofon D. Koutsoukos, Panos J. Antsaklis, James A. Stiver, and Michael D. Lemmon. Supervisory control of hybrid systems. In Proceedings of the IEEE, 2000.

[16] Ricardo G. Sanfelice and Andrew R. Teel. Dynamical properties of hybrid systems simulators. Automatica, 46, 2009.

[17] Alexandre M. Bayen, Ian M. Mitchell, Meeko M. K. Oishi, and Claire J. Tomlin. Aircraft autolander safety analysis through optimal control-based reach set computation. Journal of Guidance, Control, and Dynamics, 30(1), 2007.

[18] Bernard Etkin and Lloyd Duff Reid. Dynamics of Flight - Stability and Control. John Wiley and Sons, Inc., 1996.

[19] John D. Anderson. Fundamentals of Aerodynamics. McGraw-Hill, 1991.

[20] Ira H. Abbott and Albert E. von Doenhoff. Theory of Wing Sections. Dover Publications, Inc., 1959.

[21] Richard Von Mises. Theory of Flight. General Publishing Company, 1959.

[22] Alberto Isidori, Lorenzo Marconi, and Andrea Serrani. Robust Autonomous Guidance. Springer, 2003.

[23] Hassan K. Khalil. Nonlinear Systems. Prentice Hall, 2002.

[24] U.S. Air Force. U.s. military specification mil-f-8785c, 1980. 